\title{
AN ISOMORPHISM THEOREM FOR COMMUTATIVE MODULAR GROUP ALGEBRAS
}

\author{
WILLIAM ULLERY
}

(Communicated by Warren J. Wong)

\begin{abstract}
For each positive integer $n$ and limit ordinal $\mu$, a new class of abelian $p$-groups, called $A_{n}(\mu)$-groups, are introduced. These groups are shown to be uniquely determined up to isomorphism by numerical invariants which include, but are not restricted to, their Ulm-Kaplansky invariants. As an application of this uniqueness theorem, we prove an isomorphism result for group algebras: Let $H$ be an $A_{n}(\mu)$-group and $F$ a field of characteristic $p$. It is shown that if $K$ is a group such that the group algebras $F H$ and $F K$ are $F$-isomorphic, then $H$ and $K$ are isomorphic.
\end{abstract}

\section{INTRODUCTION}

Suppose $F$ is a field of characteristic $p>0$, and let $H$ be an abelian $p$-group. If $K$ is a group such that the group algebras $F H$ and $F K$ are $F$ isomorphic, the question of whether $H \cong K$ arises. It has been known for some time that the Ulm-Kaplansky invariants of $H$ and $K$ must be equal [1, 6]. Thus, if $H$ is countable it follows that $H \cong K$ by the well-known Ulm's Theorem for countable abelian $p$-groups.

Several generalizations of this basic result have been made over the last twenty years. In 1969, Berman and Mollov [2] showed that if $H$ is an arbitrary direct sum of cyclic $p$-groups, then $K$ must also be such a group, hence $H \cong K$. Later, this result was extended [7] to the case where $H$ is totally projective of length less than the first uncountable ordinal. More recently, the cases where $H$ is totally projective [8], an $N$-group [9, 10], and an elementary $A$-group [10] have been shown to imply $H \cong K$.

In this paper we further extend these known isomorphism results. In $\S 2$, for each positive integer $n$ and limit ordinal $\mu$, we define a class of $p$-groups, which we call $A_{n}(\mu)$-groups. In the case $n=1$ these are the $\mu$-elementary $A$-groups of Hill [4]. Moreover, the class $A_{n}(\mu)$ consists entirely of totally projective groups if and only if $\mu$ is cofinal with $\omega_{0}$ (Lemma 1). Thus, in case

Received by the editors March 13,1989 and, in revised form, January 19, 1990.

1980 Mathematics Subject Classification (1985 Revision). Primary 20K10; Secondary 20C07.

Key words and phrases. Modular group algebras, invariants for abelian $p$-groups. 
$n \geq 2$ and $\mu$ is not cofinal with $\omega_{0}$, new classes of $p$-groups are obtained (see the example following Theorem 2).

In $\S 3$ we introduce cardinal invariants for $A_{n}(\mu)$-groups and prove a result (Theorem 2) which may be of independent interest; namely, two $A_{n}(\mu)$-groups are isomorphic if and only if their associated invariants are equal. We remark that these invariants include, but are not restricted to, the Ulm-Kaplansky invariants. Finally, in the last section, we apply the uniqueness theorem of $\S 3$ to extend the group algebra isomorphism results discussed above to the case where $H$ is an $A_{n}(\mu)$-group, for any positive integer $n$ and limit ordinal $\mu$.

Throughout, $p$ is an arbitrary fixed prime and all groups considered are $p$ primary abelian groups, written multiplicatively. Abelian group terminology not explicitly defined herein is in agreement with Fuchs [3].

\section{A CLASS OF $p$-GROUPS}

If $G$ is a $p$-group and $\sigma$ is an ordinal, we write $G^{\sigma}$ for the subgroup of $G$ consisting of the elements of $G$ of heights $\geq \sigma$. If $G$ has limit length $\mu$, an isotype subgroup $H$ of $G$ is called an almost balanced subgroup of $G$ provided that $(G / H)^{\sigma}=G^{\sigma} H / H$ for every $\sigma<\mu$.

Fix a limit ordinal $\mu$. For each positive integer $n$ we define a class of p-groups $A_{n}(\mu)$ as follows. Call $H$ an $A_{1}(\mu)$-group if there exists a totally projective group $G$ of length $\mu$ such that $H$ is almost balanced in $G$ and $G / H$ is totally projective. For $n \geq 2$, call $H$ an $A_{n}(\mu)$-group if there exists a totally projective group $G$ of length $\mu$ satisfying the following conditions:

(a) $H$ is an almost balanced subgroup of $G$.

(b) $(G / H)^{\mu}$ is an $A_{n-1}(\mu)$-group.

(c) $(G / H) /(G / H)^{\mu}$ is totally projective.

If $G$ and $H$ satisfy the conditions set forth above, for some positive integer $n$, we call $(H, G)$ an $A_{n}(\mu)$-pair.

Several comments are in order. First, we do not require totally projective groups to be reduced. If desired, it is no great loss to consider only reduced $A_{n}(\mu)$-groups; however, we shall not do this. We also remark that our $A_{1}(\mu)$ groups are the $\mu$-elementary $A$-groups as defined in [4]. Thus, our introduction of the classes $A_{n}(\mu)$ may be viewed as a modest attempt to extend P. Hill's theory of $A$-groups. Indeed, our results for $A_{n}(\mu)$-groups are direct extensions of known results for $\mu$-elementary $A$-groups. Since the class of $\mu$-elementary $A$-groups consists entirely of totally projective groups if and only if $\mu$ is cofinal with $\omega_{0}[4$, Theorem 1], a routine induction yields our first result.

Lemma 1. For each positive integer $n$, the class $A_{n}(\mu)$ consists entirely of totally projective groups if and only if $\mu$ is cofinal with $\omega_{0}$.

In view of Lemma 1 , we restrict our attention to the classes $A_{n}(\mu)$ where $\mu$ is not cofinal with $\omega_{0}$ (this means that $\mu$, though a limit, is not the limit of a countable sequence of smaller ordinals). 


\section{INVARIANTS AND A UNIQUENESS THEOREM}

Suppose $(H, G)$ is an $A_{n}(\mu)$-pair where the limit ordinal $\mu$ is not cofinal with $\omega_{0}$. It is well known that $G$ is Hausdorff and complete in its $\mu$-topology (the topology obtained by taking the subgroups $G^{\sigma}(\sigma<\mu)$ as the neighborhoods of the identity). Denoting by $\bar{H}$ the closure of $H$ in $G$, observe $(G / H)^{\mu}=$ $\bar{H} / H$.

Throughout we adjoin $\infty$ to the ordinals with the convention $\sigma<\infty$ for every ordinal $\sigma$. For any $p$-group $A$, we denote by $f_{A}$ the extended UlmKaplansky function of $A$. That is, for every ordinal $\sigma \leq \infty$,

$$
f_{A}(\sigma)= \begin{cases}\operatorname{dim}\left(A^{\sigma}[p] / A^{\sigma+1}[p]\right), & \text { if } \sigma<\infty, \\ \operatorname{dim}\left(A^{\infty}[p]\right), & \text { if } \sigma=\infty .\end{cases}
$$

Here $A^{\infty}$ is simply the divisible part of $A$ and it is understood that dimensions are computed over the field with $p$ elements.

With the above notation, for each positive integer $n$ we define a cardinalvalued function $f_{n}^{H}$ of $n+1$ ordinal variables as follows:

$$
f_{1}^{H}\left(\alpha_{1}, \alpha_{2}\right)= \begin{cases}f_{H}\left(\alpha_{2}\right), & \text { if } \alpha_{1}=0 \\ f_{\bar{H} / H}\left(\alpha_{2}\right), & \text { if } \alpha_{1}=\mu \\ 0, & \text { otherwise }\end{cases}
$$

For $n \geq 2$, we define

$$
f_{n}^{H}\left(\alpha_{1}, \alpha_{2}, \ldots, \alpha_{n+1}\right)= \begin{cases}f_{H}\left(\alpha_{n+1}\right), & \text { if } \alpha_{1}=\cdots=\alpha_{n}=0, \\ f_{n-1}^{\bar{H} / H}\left(\alpha_{2}, \ldots, \alpha_{n+1}\right), & \text { if } \alpha_{1}=\mu, \\ 0, & \text { otherwise. }\end{cases}
$$

We refer to the function $f_{n}^{H}$ as the $A_{n}(\mu)$-invariant of $H$. Since $\bar{H}$ may be viewed as the completion of $H$ in its $\mu$-topology, it is clear that each $f_{n}^{H}$ is an isomorphism invariant of $H$, independent of the choice of a containing totally projective group $G$.

Theorem 2. Suppose $\mu$ is a limit ordinal not cofinal with $\omega_{0}$ and $n$ is a positive integer. Two $A_{n}(\mu)$-groups $H$ and $K$ are isomorphic if and only if they have the same $A_{n}(\mu)$-invariants.

Proof. In view of our observations above, we need only show that $H \cong K$ provided that $f_{n}^{H}=f_{n}^{K}$. Since the case $n=1$ is the known result for $\mu$ elementary $A$-groups [4, Theorem 3], we may assume $n \geq 2$ and proceed by induction on $n$.

Select totally projective groups $G$ and $T$, each of length $\mu$, such that $(H, G)$ and $(K, T)$ are $A_{n}(\mu)$-pairs. Since $(G / H)^{\mu}$ and $(T / K)^{\mu}$ are $A_{n-1}(\mu)$-groups with the same $A_{n-1}(\mu)$-invariants, the induction hypothesis implies $(G / H)^{\mu} \cong$ $(T / K)^{\mu}$. Set

$$
A=\oplus_{\aleph_{0}}\left\{G \oplus T \oplus(G / H) /(G / H)^{\mu} \oplus(T / K) /(T / K)^{\mu}\right\},
$$


and let $L=G \oplus T \oplus A$, a totally projective group of length $\mu$. Identifying $G$ (respectively, $T$ ) with the first (respectively, second) direct factor of $L$, note that

$$
(L / H) /(L / H)^{\mu} \cong(L / K) /(L / K)^{\mu}
$$

and that both of these groups are totally projective. In view of the isomorphism $(G / H)^{\mu} \cong(T / K)^{\mu}$, we have $(L / H)^{\mu} \cong(L / K)^{\mu}$. Thus, this latter isomorphism extends to an isomorphism $L / H \cong L / K$, by virtue of Zippin's Theorem for totally projective groups. Note $f_{n}^{H}=f_{n}^{K}$ includes equality of the Ulm-Kaplansky invariants $f_{H}$ and $f_{K}$. Therefore, since $H$ and $K$ are almost balanced subgroups of $L$, the main theorem in [5] implies $H \cong K$.

To see that Theorem 2 actually gives us something new, the following example shows that there exist $A_{2}(\mu)$-groups which are not $\mu$-elementary $A$-groups. The reference for the theory of $c$-valuations used below is [5].

Example. Let $\mu$ be a limit ordinal not cofinal with $\omega_{0}$, and let $E$ be a $\mu$ elementary $A$-group which is not totally projective. Select a $p$-group $A$ such that $A^{\mu} \cong E$ and $A / A^{\mu}$ is totally projective. Such an $A$ may be seen to exist, for example, by adapting the portion of the proof of Theorem 4 in [4, p. 516]. Define a $c$-valuation on $A$ by

$$
\|a\|= \begin{cases}\alpha+1, & \text { if } \alpha<\mu \text { and } a \in A^{\alpha} \backslash A^{\alpha+1}, \\ \mu, & \text { if } a \in A^{\mu} \text { and } a \neq 0, \\ \infty, & \text { if } a=0,\end{cases}
$$

for every $a \in A$. By Theorem 2.8 in [5], there exists a totally projective group $G$ and an isotype subgroup $H$ of $G$ such that $G / H \cong A$ as $c$-valuated groups, where the $c$-valuation on $G / H$ is the coset valuation. It now easily follows that $(H, G)$ is an $A_{2}(\mu)$-pair. Moreover, $H$ is not an $A_{1}(\mu)$-group, since otherwise $\bar{H} / H \cong E$ would be totally projective.

\section{AN APPLiCATION TO MODUlaR GROUP ALGEBRAS}

Suppose $F$ is a field of characteristic $p>0$ and $H$ and $K$ are $p$-groups such that the group algebras $F H$ and $F K$ are $F$-isomorphic. As an application of Theorem 2, we prove that if $H$ (but not a priori $K$ ) is an $A_{n}(\mu)$-group, for some positive integer $n$ and limit ordinal $\mu$, then $H \cong K$.

Suppose $A$ is a $p$-group. Letting aug: $F A \rightarrow F$ denote the augmentation map, we set $I(F A)=\{x \in F A: \operatorname{aug}(x)=0\}$ and $V(F A)=\{x \in F A: \operatorname{aug}(x)=$ $1\}$. Note that $V(F A)$ is a $p$-group and a subgroup of the group of units of $F A$. Moreover, if $H$ is a subgroup of $V(F A)$ and $V(F A) /(1+F A \cdot I(F H)) \cong$ $V(F(A / H))$. As a direct corollary of Lemmas 1 and 2 in the author's paper [9], we obtain the following result.

Lemma 3. Suppose $F$ is a perfect field of characteristic $p>0$. If $H$ is $a$ subgroup of a p-group $A$ of limit length $\mu$, then $V(F A)$ has length $\mu$ and $H$ 
is almost balanced in $A$ if and only if $1+F A \cdot I(F H)$ is almost balanced in $V(F A)$.

We are now in position to prove our isomorphism result. We state a slightly more general version than is indicated above. As we shall see, this causes no difficulties in the proof.

Theorem 4. Suppose $R$ is a commutative ring with 1 and $p$ is a prime number such that $p \cdot 1$ is not a unit in $R$. Suppose further that $H$ is a p-group and $K$ is an abelian group with $R H \cong R K$ as $R$-algebras. Then, if $H$ is an $A_{n}(\mu)$-group for some positive integer $n$ and limit ordinal $\mu, H \cong K$.

Proof. Let $M$ be a maximal ideal of $R$ containing $p$, and let $F$ be an algebraic closure of $R / M$. Consequently, $F$ is a perfect field of characteristic $p$ and $F H \cong F K$. Since there is an augmentation-preserving isomorphism $F K \rightarrow$ $F H, V(F H)$ contains an $F$-basis for $F H$ isomorphic to $K$. Therefore, we may assume $K \leq V(F H), F H=F K$ and $I(F H)=I(F K)$.

We now proceed by induction on $n$. Since the case $n=1$ is the theorem in [10], we may assume $n \geq 2$. Moreover, since $H \cong K$ whenever $H$ is totally projective by $[8$, Corollary to Theorem 3$]$, we may assume $\mu$ is not cofinal with $\omega_{0}$ by Lemma 1.

Let $G$ be a totally projective group of length $\mu$ such that $(H, G)$ is an $A_{n}(\mu)$-pair. By Theorems 1 and 2 in [8], $A=V(F G)$ is totally projective of length $\mu$ and contains $G$ as a direct factor. Thus, $(H, A)$ is an $A_{n}(\mu)$-pair and $1+F A \cdot I(F H)=1+F A \cdot I(F K)$ is almost balanced in $V(F A)$ by Lemma 3. Thus, a further application of Lemma 3 shows that $K$ is almost balanced in $A$. Note that $F(A / H) \cong F A / F A \cdot I(F H)=F A / F A \cdot I(F K) \cong F(A / K)$. Thus, $F$ perfect implies $F\left((A / H)^{\mu}\right) \cong F\left((A / K)^{\mu}\right)$ and, since $A /(H)^{\mu}$ is an $A_{n-1}(\mu)$-group, the induction hypothesis implies $(A / H)^{\mu} \cong(A / K)^{\mu}$. Moreover, $f_{H}=f_{K}$ by [6, Proposition 5]. Therefore, if $K$ were an $A_{n}(\mu)$-group, $H$ and $K$ would have the same $A_{n}(\mu)$-invariants. Thus, in view of Theorem 2 , the proof will be complete once we have shown that $K$ is an $A_{n}(\mu)$-group.

As observed above, $K$ is almost balanced in $A$ and $(A / K)^{\mu} \cong(A / H)^{\mu}$ is an $A_{n-1}(\mu)$-group. Moreover, the isomorphism $F(A / H) \rightarrow F(A / K)$ may be assumed augmentation-preserving. Any such isomorphism restricts to an isomorphism of $F\left((A / H)^{\mu}\right)$ onto $F\left((A / K)^{\mu}\right)$ and maps $I\left(F(A / H)^{\mu}\right)$ onto $I\left(F(A / K)^{\mu}\right)$. Therefore, $F\left((A / H) /(A / H)^{\mu}\right) \cong F(A / H) /\left(F(A / H) \cdot I\left(F(A / H)^{\mu}\right)\right)$ $\cong F(A / K) /\left(F(A / K) \cdot I\left(F(A / K)^{\mu}\right)\right) \cong F\left((A / K) /(A / K)^{\mu}\right)$. Since $(A / H) /(A / H)^{\mu}$ is totally projective, a further application of the corollary to Theorem 3 in [8] implies $(A / K) /(A / K)^{\mu}$ is totally projective. Therefore, $(K, A)$ is an $A_{n}(\mu)$ pair, and the proof is complete.

\section{REFERENCES}

1. S. D. Berman, Group algebras of countable abelian p-groups, Publ. Math. Debrecen 14 (1967), 365-405.

2. S. D. Berman and T. Zh. Mollov, The group rings of abelian p-groups of arbitrary power, Mat. Zametki 6 (1969), 381-392; Math. Notes 6 (1969), 686-692. (Russian) 
3. L. Fuchs, Infinite abelian groups, vol. II. Academic Press, New York, 1973.

4. P. Hill, On the structure of abelian p-groups, Trans. Amer. Math. Soc. 288 (1985), 505-525.

5. P. Hill and C. Megibben, On the theory and classification of abelian p-groups, Math. Z. 190 (1985), 17-38.

6. W. May, Commutative group algebras, Trans. Amer. Math. Soc. 136 (1969), 139-149.

7. __. Modular group algebras of totally projective p-primary groups, Proc. Amer. Math. Soc. 76 (1979), 31-34.

8. __ Modular group algebras of simply presented abelian groups, Proc. Amer. Math. Soc. 104 (1988), 403-409.

9. W. Ullery, Modular group algebras of N-groups, Proc. Amer. Math. Soc. 103 (1988), 10531057.

10. _ Modular group algebras of isotype subgroups of totally projective p-groups, Comm. Algebra (to appear).

Department of Mathematics, Auburn University, Auburn, Alabama 36849 